\title{
AUTOMORPHISMS OF A FINITE ABELIAN GROUP WHICH REDUCE TO THE IDENTITY ON A SUBGROUP OR FACTOR GROUP
}

\author{
J. C. HOWARTH
}

1. The automorphism group of any group $G$ we denote by $\Gamma(G)$. We shall consider the following two types of subgroups of $\Gamma(G)$ : if $H$ is a subgroup of $G, \Gamma(G: H)$ is the subgroup consisting of all automorphisms which are the identity on $H$, and if $H$ is a normal subgroup, $\Gamma(G: G / H)$ is the subgroup consisting of all automorphisms which leave $H$ invariant and which induce the identity on $G / H$.

For the special case where $G$ is finite Abelian, it is the connection between these two types of groups which is to be studied in this paper. Since any finite Abelian group is the direct sum of $p$-groups, each of which is a characteristic subgroup, it will then be sufficient to consider only the case of a $p$-group, for some fixed prime $p$. We will write the group additively. The order of any element $g$ of a finite group $G$ we denote by $|g|$. All operators are to be written on the right.

It may perhaps be mentioned here that these subgroups of automorphisms of an Abelian group do arise in connection with the theory of the structure of the automorphism group of any finite group $G$. In fact, it has been shown by Green [3], that if $G$ has centre $Z$ and derived group $G^{\prime}$ then, writing $\Pi=G / Z$, there exists an Abelian group $Q$ containing an isomorphic copy of $Z$ with $Q / Z \cong \Pi / \Pi^{\prime}$, such that

$$
\Gamma(G: G / Z) \cong \Gamma(Q: Q / Z) \cap \Gamma\left(Q: G^{\prime} \cap Z\right) .
$$

I am indebted to Dr. W. Ledermann for the many useful suggestions he has made concerning this subject, during my period as a research student at Manchester University.

2. Let an Abelian $p$-group $A$ have basis elements $a_{1}, \cdots, a_{r}$, with orders $p^{m(1)}, \cdots, p^{m(r)}$ respectively.

Denote the character group of $A$ by $A^{*}$. It is shown in Fricke [2, pp. 317-324] that a choice of dual bases in $A$ and $A^{*}$ yields an isomorphism of $A$ with $A^{*}$. That is, if we define

$$
\left(a_{k}\right) \chi_{j}=\left\{\begin{array}{cl}
1 & \text { if } j \neq k, \\
\exp 2 \pi i p^{-m(k)} & \text { if } j=k,
\end{array}\right.
$$

then the mapping $a \mu=\left(\sum \alpha_{j} a_{j}\right) \mu=\prod \chi_{j}^{\alpha_{j}}$, is an isomorphism. We shall sometimes denote $a \mu$ by $\chi_{a}$.

Received by the editors October 26, 1959 and, in revised form, June 3, 1960. 
If $\theta$ is an automorphism of $A$ then $\theta$ induces an automorphism $\theta^{*}$ of $A^{*}$ by the rule $a\left(\chi \theta^{*}\right)=(a \theta) \chi$. In this case $\theta \rightarrow \theta^{*}$ is an anti-isomorphism of $\Gamma(A)$ onto $\Gamma\left(A^{*}\right)$, for if $\theta_{1}, \theta_{2} \in \Gamma(A)$ and $\chi \in A^{*}$ then

$$
a\left(\chi \theta_{1}^{*} \theta_{2}^{*}\right)=\left(a \theta_{2}\right)\left(\chi \theta_{1}^{*}\right)=a\left(\theta_{2} \theta_{1}\right) \chi=a\left(\chi\left(\theta_{2} \theta_{1}\right)^{*}\right) .
$$

3. Assume now that $B$ is a given subgroup of $A$. Important for our present purposes is the following result, see Fricke [2, p. 322].

Lemma 3.1. The set of all characters $\chi$ such that $\chi(b)=1$ for all elements $b$ of $B$, forms a subgroup $B^{\prime}$ of $A^{*}$ which is isomorphic to $A / B$.

We are now in a position to give our first result concerning the groups $\Gamma(A: B)$ and $\Gamma(A: A / B)$.

Theorem 3.2. An automorphism $\gamma$ of $A$ belongs to $\Gamma(A: B)$ if and only if $\gamma^{*}$ lies in $\Gamma\left(A^{*}: A^{*} / B^{\prime}\right)$.

Proof. Suppose first that $\gamma$ is an element of $\Gamma(A: B)$, then $b \gamma=b$ for any element $b$ of $B$. Thus, for any character $\chi$,

$$
b\left(\chi \gamma^{*} \cdot \chi^{-1}\right)=b\left(\chi \gamma^{*}\right) \cdot b \chi^{-1}=(b \gamma) \chi \cdot b \chi^{-1}=b \chi \cdot b \chi^{-1}=1
$$

and therefore $\chi \gamma^{*} \cdot \chi^{-1}$ lies in $B^{\prime}$. Hence $\chi \gamma^{*}=\chi \cdot \chi^{\prime}$ for some element $\chi^{\prime}$ of $B^{\prime}$, and so $\gamma^{*}$ belongs to $\Gamma\left(A^{*}: A^{*} / B^{\prime}\right)$.

If, on the other hand, $\gamma$ is an automorphism of $A$ having $\gamma^{*}$ belonging to $\Gamma\left(A^{*}: A^{*} / B^{\prime}\right)$, then, for any element $b$ of $B$ and character $\chi$,

$$
(b \gamma-b) \chi=(b \gamma) \chi \cdot(-b) \chi=b\left(\chi \gamma^{*}\right) \cdot b \chi^{-1}=b\left(\chi \gamma^{*} \cdot \chi^{-1}\right)=1 \text {. }
$$

Thus, every character $\chi$ maps each element $b \gamma-b$ into unity and therefore, by Lemma $3.1, b \gamma-b=0$ identically, and so each $\gamma$ lies in $\Gamma(A: B)$, which completes the proof of the theorem.

We now state a dual version of this result, for which a proof may be constructed on similar lines.

Theorem 3.3. An automorphism $\gamma$ of $A$ lies in $\Gamma(A: A / B)$ if and only if $\gamma^{*}$ belongs to $\Gamma\left(A^{*}: B^{\prime}\right)$.

4. The results of $\S 3$ may be presented in an alternative form, by making use of the isomorphism $\mu$ of $\$ 2$.

If, for the subgroup $B$ of $A$ we write $B^{0}=B^{\prime} \mu^{-1}$ then $B^{0}$ is a subgroup of $A$ which, by Lemma 3.1, is isomorphic to $A / B$. An element $b^{0}=\beta_{1}^{0} a_{1}+\cdots+\beta_{r}^{0} a_{r}$ of $A$ belongs to $B^{0}$ if and only if the character $\chi_{b^{0}}=b^{0} \mu$ lies in $B^{\prime}$. By definition, we have, for $j=1, \cdots, r$,

$$
a_{j} \chi_{b 0}=\exp \left(2 \pi i \beta_{j}^{0} p^{-m(j)}\right)
$$


and so $\chi_{b 0}$ lies in $B^{\prime}$ if and only if, for each element $b=\beta_{1} a_{1}+\cdots$ $+\beta_{r} a_{r}$ of $B$,

$$
b X_{b 0}=\exp \left(2 \pi i \beta_{1}^{0} \beta_{1} p^{-m(1)}\right) \cdots \exp \left(2 \pi i \beta_{r}^{0} \beta_{r} p^{-m(r)}\right)=1 .
$$

Thus if we say that two elements $a=\alpha_{1} a_{1}+\cdots+\alpha_{r} a_{r}$ and $a^{0}=\alpha_{1}^{0} a_{1}+\cdots+\alpha_{1}^{0} a_{r}$ are orthogonal when the condition

$$
\sum_{i=1}^{r} p^{m(1)-m(i)} \alpha_{i} \alpha_{i}^{0} \equiv 0\left(\bmod p^{m(1)}\right)
$$

is satisfied, we have by Lemma 3.1, the following result (cf. Birkhoff $[1$, p. 389]).

Lemma 4.1. If $B$ is a subgroup of $A$ then the set of those elements of $A$ which are orthogonal to each element of $B$ coincides with the subgroup $B^{0}$ of $A$ isomorphic to $A / B$.

Corollary 4.2. If $B$ is a subgroup of $A$ then $B^{00}=B$.

Proof. It is clear from the definition of orthogonality that $B^{00} \supseteq B$, but since $B^{00}$ is isomorphic to $A / B^{0}$, it follows that $B^{00}$ has the same order as $B$, which proves the result.

It was shown in $\$ 2$ that if $\theta$ is any automorphism of $A$ then we may define an automorphism $\theta^{*}$ of $A^{*}$ by the rule

$$
a\left(\chi \theta^{*}\right)=(a \theta) \chi
$$

and furthermore, the correspondence $\theta \rightarrow \theta^{*}$ is an anti-isomorphism of $\Gamma(A)$ onto $\Gamma\left(A^{*}\right)$. Hence, since $\mu$ is an isomorphism, the mapping $\theta \rightarrow \theta^{0}$, where $\theta^{0}=\mu \theta^{*} \mu^{-1}$, is an anti-automorphism of the group $\Gamma(A)$. We now establish a result analogous to Theorem 3.2.

Theorem 4.3. An automorphism $\gamma$ of $A$ belongs to $\Gamma(A: B)$ if and only if $\gamma^{0}=\mu \gamma^{*} \mu^{-1}$ lies in $\Gamma\left(A: A / B^{0}\right)$.

Proof. Suppose first that $\gamma$ lies in $\Gamma(A: B)$ then, by Theorem 3.2, $\gamma^{*}$ belongs to $\Gamma\left(A^{*}: A^{*} / B^{\prime}\right)$ that is, for any character $\chi_{a}$ we have $\chi_{a} \gamma^{*}=\chi_{a} \cdot \chi^{\prime}$ where $\chi^{\prime}$ is an element of $B^{\prime}$. Now $B^{\prime}=B^{0} \mu$, therefore for some element $b^{0}$ of $B^{0}, \chi_{a} \gamma^{*}=\chi_{a} \chi_{b^{0}}=\chi_{a+b 0}$. Thus, for any element $a$ of $A$,

$$
a \gamma^{0}=a \mu \gamma^{*} \mu^{-1}=\chi_{a} \gamma^{*} \mu^{-1}=\chi_{a+b^{0} \mu^{-1}}=a+b^{0}
$$

and hence $\gamma^{0}$ is an element of $\Gamma\left(A: A / B^{0}\right)$.

On the other hand, if $\gamma$ is an automorphism of $A$ such that $\mu \gamma^{*} \mu^{-1}$ lies in $\Gamma\left(A: A / B^{0}\right)$ then, for any element $a$ of $A$ there exists an element $b^{0}$ of $B^{0}$ with $a \mu \gamma^{*} \mu^{-1}=a+b^{0}$. Applying $\mu$ to each side, we have 
$a \mu \gamma^{*}=\chi_{a} \gamma^{*}=\left(a+b^{0}\right) \mu=\chi_{a} \chi_{b 0}=\chi_{b} \chi_{a}$, and therefore $\chi_{a} \gamma^{*} \chi_{a}^{-1}=\chi_{b^{0}}$ is an element of $B^{\prime}$. Thus $\gamma^{*}$ lies in $\Gamma\left(A^{*}: A^{*} / B^{\prime}\right)$ and hence, by Theorem 3.2, $\gamma$ is an element of $\Gamma(A: B)$, which completes the proof.

COROLlary 4.4. There is an automorphism of the group $\Gamma(A)$ which maps $\Gamma(A: B)$ onto $\Gamma\left(A: A / B^{0}\right)$.

Replacing $B$ by $B^{0}$ in these results and using Corollary 4.2 would yield dual versions. (Cf. Theorem 3.3.)

5. If we suppose that an automorphism $\theta$ of $A$ corresponds to a matrix $S=\left(\theta_{i j}\right)$ so that, for $i=1, \cdots, r$,

$$
a_{i} \theta=\sum_{j=1}^{r} \theta_{i j} a_{j},
$$

we now determine the matrix, say $S^{0}=\left(\theta_{4 j}^{0}\right)$, of the coefficients of the automorphism $\theta^{0}$. It is to be shown that $S^{0}$ is a form of transpose of the matrix $S$, and that $S^{00}=S$, from which follows the identity $\theta^{00}=\theta$. We note first that since $\left|a_{i} \theta\right|=\left|a_{i}\right|$ then, for $i>j, \theta_{i j}$ is divisible by $p^{m(j)-m(i)}$.

As before, let $\chi_{1}, \cdots, \chi_{r}$ be the basis of $A^{*}$ which is the image of the basis $a_{1}, \cdots, a_{r}$, of $A$ under the isomorphism $\mu$, then for integers $j$ and $k$ in the range $1 \leqq j, k \leqq r$,

$$
\begin{aligned}
a_{k}\left(\chi_{j} \theta^{*}\right) & =\left(a_{k} \theta\right) \chi_{j}=\left(\sum_{l=1}^{r} \theta_{k l} a_{l}\right) \chi_{j}=\prod_{l=1}^{r}\left(a_{l} \chi_{j}\right)^{\theta_{k l}}=\left\{a_{j} \chi_{j}\right\}^{o_{k j}} \\
& =\exp \left(2 \pi i \theta_{k j} p^{-m(j)}\right) .
\end{aligned}
$$

Consider now the character, say $\chi_{j}^{\prime}$, defined by

$$
\chi_{j}^{\prime}=\prod_{l=1}^{r} \chi_{l}^{p^{m(l)-m(j) o_{l j}}}
$$

We have, for each $k$,

$$
a_{k} \chi_{j}^{\prime}=\left\{\exp \left(2 \pi i p^{-m(k)}\right)\right\}^{m(k)-m(j) o_{k j}}=\exp \left(2 \pi i \theta_{k j} p^{-m(j)}\right)
$$

and thus it is seen that $\chi_{j} \theta^{*}=\chi_{j}^{\prime}$. Hence it follows that, for $i, j$ $=1, \cdots, r, \theta_{i j}^{0}=p^{m(j)-m(i)} \theta_{j i}$. That is, if

$$
S=\left[\begin{array}{ccc}
\theta_{11} & \theta_{12} & \theta_{1 r} \\
p^{m(1)-m(2)} \theta_{21}^{\prime} & \theta_{22} & \theta_{2 r} \\
p^{m(1)-m(r)} \theta_{r 1}^{\prime} & p^{m(2)-m(r)} \theta_{r 2}^{\prime} & \theta_{r r}
\end{array}\right]
$$

then 


$$
S^{0}=\left[\begin{array}{ccc}
\theta_{11} & \theta_{21}^{\prime} & \theta_{r 1}^{\prime} \\
p^{m(1)-m(2)} \theta_{12} & \theta_{22} & \theta_{r 2}^{\prime} \\
p^{m(1)-m(r)} \theta_{1 r} & p^{m(2)-m(r)} \theta_{2 r} & \theta_{r r}
\end{array}\right] .
$$

This construction shows immediately that $\left(S^{0}\right)^{0}=S$ and therefore, for any automorphism $\theta, \theta^{00}=\theta$. If $\Gamma$ is any subgroup of $\Gamma(A)$, denote by $\Gamma^{0}$ the set $\Gamma^{0}=\left\{\gamma^{0} \mid \gamma \in \Gamma\right\}$ then the groups $\Gamma$ and $\Gamma^{0}$ can be mapped onto one another by automorphisms of $\Gamma(A)$. Also, since $\gamma^{00}=\gamma$ we have $\Gamma^{00}=\Gamma$. We now restate the result of Theorem 4.3.

Theorem 5.1. $\Gamma(A: B)=\Gamma^{0}\left(A: A / B^{0}\right)$.

Three equivalent versions of this may be obtained by using the identities $B^{00}=B$ and $\Gamma^{00}=\Gamma$.

6. If $B_{1}$ and $B_{2}$ are isomorphic subgroups of $A$, then the groups $\Gamma\left(A: B_{1}\right)$ and $\Gamma\left(A: B_{2}\right)$ are not necessarily isomorphic. However, we give a positive result in this connection.

THEOREM 6.1. If $B$ is a subgroup of $A$, then for any automorphism $\gamma$ of $A, \Gamma(A: B \gamma) \cong \Gamma(A: B)$.

Proof. Suppose that an automorphism $\theta$ of $A$ belongs to $\Gamma(A: B)$, then for any element $b \gamma$ of $B \gamma$ we have

$$
b \gamma \cdot \gamma^{-1} \theta \gamma=b \theta \gamma=b \gamma \text {. }
$$

Thus $\gamma^{-1} \theta \gamma$ lies in $\Gamma(A: B \gamma)$ and hence $\Gamma(A: B \gamma)$ contains a subgroup isomorphic to $\Gamma(A: B)$. The result now follows by symmetry.

For the corresponding result with groups of the type $\Gamma(A: A / B)$ we use the following lemma.

LEMMa 6.2.

$$
(B \gamma)^{0}=B^{0}\left(\gamma^{0}\right)^{-1} .
$$

Proof. It is worthwhile mentioning, first of all, that for any automorphism $\gamma,\left(\gamma^{0}\right)^{-1}=\left(\gamma^{-1}\right)^{0}$. This follows from the fact that the correspondence $\gamma \rightarrow \gamma^{0}$ is an anti-automorphism of the group $\Gamma(A)$, for we then have $\left(\gamma^{-1}\right)^{0} \cdot \gamma^{0}=\left(\gamma \cdot \gamma^{-1}\right)^{0}=\iota^{0}=\imath$, where $\iota$ denotes the identity automorphism. It may be shown, in a similar way, that $\left(\gamma^{*}\right)^{-1}$ $=\left(\boldsymbol{\gamma}^{-1}\right)^{*}$.

By definition, we have $(B \gamma)^{0}=(B \gamma)^{\prime} \mu^{-1}$ and we first of all show that $(B \gamma)^{\prime}=B^{\prime} \gamma^{*-1}$. Suppose that a character $\chi$ is a member of the subgroup $B^{\prime} \gamma^{*-1}$ then for some character $\chi^{\prime}$ belonging to $B^{\prime}, \chi=\chi^{\prime} \gamma^{-1 *}$ and therefore, for any element $a$ of $A, a \chi=\left(a \gamma^{-1}\right) \chi^{\prime}$. In particular, 
for any element $b$ of $B$ we have, since $\chi^{\prime}$ belongs to $B^{\prime}$,

$$
(b \gamma) \chi=\left(b \gamma \cdot \gamma^{-1}\right) \chi^{\prime}=b \chi^{\prime}=1
$$

and therefore $\chi$ lies in $(B \gamma)^{\prime}$. Thus we have shown that $(B \gamma)^{\prime} \supseteq B^{\prime} \gamma^{-1 *}$, but the two groups have the same order and therefore they coincide. Consequently, $(B \gamma)^{0}=(B \gamma)^{\prime} \mu^{-1}=B^{\prime} \gamma^{-1 *} \mu^{-1}=B^{\prime} \mu^{-1} \cdot \mu \gamma^{-1 *} \mu^{-1}$, but by definition, $B^{0}=B^{\prime} \mu^{-1}$ and $\left(\gamma^{-1}\right)^{0}=\mu \gamma^{-1 * \mu^{-1}}$ and hence $(B \gamma)^{0}=B^{0} \gamma^{0^{-1}}$ $=B^{0}\left(\gamma^{-1}\right)^{0}$, which completes the proof.

THEOREM 6.3. If $B$ is a subgroup and $\gamma$ an automorphism of $A$, then $\Gamma(A: A / B \gamma) \cong \Gamma(A: A / B)$.

Proof. We have, using Theorem 6.1, $\Gamma(A: A / B \gamma) \cong \Gamma\left(A:(B \gamma)^{0}\right)=\Gamma\left(A: B^{0} \gamma^{0^{-1}}\right) \cong \Gamma\left(A: B^{0}\right) \cong \Gamma(A: A / B)$.

\section{REFERENCES}

1. G. Birkhoff, Subgroups of Abelian groups, Proc. London Math. Soc. (Ser. II) vol. 38 (1934) pp. 385-400.

2. R. Fricke, Lehrbuch der Algebra. I, Braunschweig, F. Vieweg, 1924.

3. J. A. Green, On the number of automorphisms of a finite group, Proc. Roy. Soc. London Ser. A vol. 237 (1956) pp. 574-581.

4. A. Ranum, The group of isomorphisms of any Abelian group, Trans. Amer. Math. Soc. vol. 8 (1907) pp. 71-91.

The University, Glasgow, Scotland 